\title{
Highway lane merge for autonomous vehicles without an acceleration area using optimal Model Predictive Control
}

\author{
Syed A. Hussain ${ }^{1}$, Babak Shahian-Jahromi, Burak Karakas and Sabri Cetin
}

\begin{abstract}
A self-driving vehicle is required to perform all lane merge scenarios when performing lane change/merge functions during its autonomous mode. Most challenging of such merge scenarios is the highway merge from a local lane to the highway lane. This is compounded when there is no acceleration or surplus road to implement the merge from the merge lane to highway lane. This paper presents a Model Predictive Control (MPC) based optimal control strategy to solve the merge problem for autonomous vehicle performing this merge. A kinematic model is used to predict the merge vehicle states. This model is then used to solve an optimal highway merge problem without an acceleration area for the merge vehicle subjected to various constraints. Simulation results are presented that show that the show the merge maneuver being implemented.
\end{abstract}

Index Terms-Highway lane merge, Autonomous, Self-driving, Model Predictive Control.

\section{INTRODUCTION}

Completely autonomous self-driving vehicles will be part of the daily commute by the end of this decade. As of 2017, there are more than 30 companies that have been granted permission to test self-driving cars on United States roads (Fig. 1) and in Europe. As more original equipment manufacturers (OEMs) get involved, many of the vehicles functionalities are being automated. These functionalities include but not limited to warning systems like lane departure, driver alertness, pedestrian detection, etc., and active control like adaptive cruise control, stop-n-go, self-parking, lane-change, and construction zone travel.

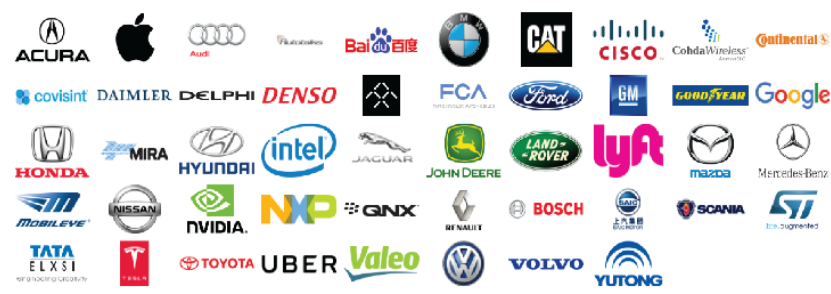

Figure 1: OEMS contributing to autonomous vehicles.

Syed A. Hussain, Electrical and Computer Engg., University of Illinois at Chicago (UIC), Chicago, USA, (e-mail: shussa50@uic.edu)

Babak Shahian-Jahromi, Mechanical and Industrial Engg., University of Illinois at Chicago (UIC), Chicago, USA, (e-mail: bshahi2@uic.edu).

Burak Karakas, Mechanical and Industrial Engg., University of Illinois at Chicago (UIC), Chicago, USA, (e-mail: bkarak2@ uic.edu)

Sabri Cetin, Mechanical and Industrial Engg., University of Illinois at Chicago (UIC), Chicago, USA, (e-mail: scetin@uic.edu)
Among the automated functions performed without the involvement of the driver, the most common and critical maneuver involve the lane merge, which could be from the one lane to another on a highway or from a local lane to the highway lane. These automated functions are a part of vehicle driver assistance systems.

Early driver assistance systems were based on sensors that measure the internal status of the vehicles. These sensors enable the control of vehicle dynamics so that the trajectory requested by the driver is followed in the best way possible. In 1995, additional dynamic driving control systems such as electronic stability control (ESC) was introduced. The second generation of driver assistance systems was introduced around the 1990s based on sensors that measure the external state of the vehicle with the focus of providing information and warnings to the driver [1].

As of today, Advanced Driver Assist Systems (ADAS) enhances the driver functions and in some cases, overrides the driver control of the vehicle. Sensors that measure conditions outside the vehicle, and vehicle position relative to its environment are essential in driving assist systems and autonomous vehicle technologies. These include: vision (camera), lidar, radar, ultrasonic range, and GPS. Current autonomous vehicles use multi-sensor platforms in which different sensors complement each other in object detection and classification.

Embedded control is increasing challenged where it seeks to combine the various real-time sensor data using sensor fusion algorithms. Low level embedded control research is very well saturated that take care of functions like acceleration, braking and steering. An important aspect of a connected vehicle is the vehicle communication which is an adhoc network between the vehicle and various road entities like vehicle, structures, pedestrian coined as Vehicle two infrastructures (V2X). These sensor fusion techniques and connectivity with other vehicles and structures on the road has enabled the autonomous vehicles to implement complex longitudinal and lateral maneuvers. These include collision avoidance, low speed stop-and-go, automated highway driving, cooperative maneuvering, etc. The key technical challenge is the embedded control software development that uses data fusion from sensors, inter-vehicular communication and real-time cloud computing to implement autonomy in the on-road vehicle to improve its safety.

The on-road vehicle automation requires a standard set of regulations and terminology with a taxonomy and definitions. Some regulation and standard have been released [2], [3]. The new standard J3016 from SAE International simplifies communication and facilitates collaboration within technical and policy domains. According to the standard as 
shown in Fig. 2, the levels of driving automation can be divided into Conditional, High, and Full Automation. The standard does not provide complete definitions applicable to lower levels of automation (No Automation, Assisted, or Partial Automation). Active safety and driver assistance system that intervene to avoid and/or mitigate an emergency and then immediately disengage are also not included for the various levels of automation.

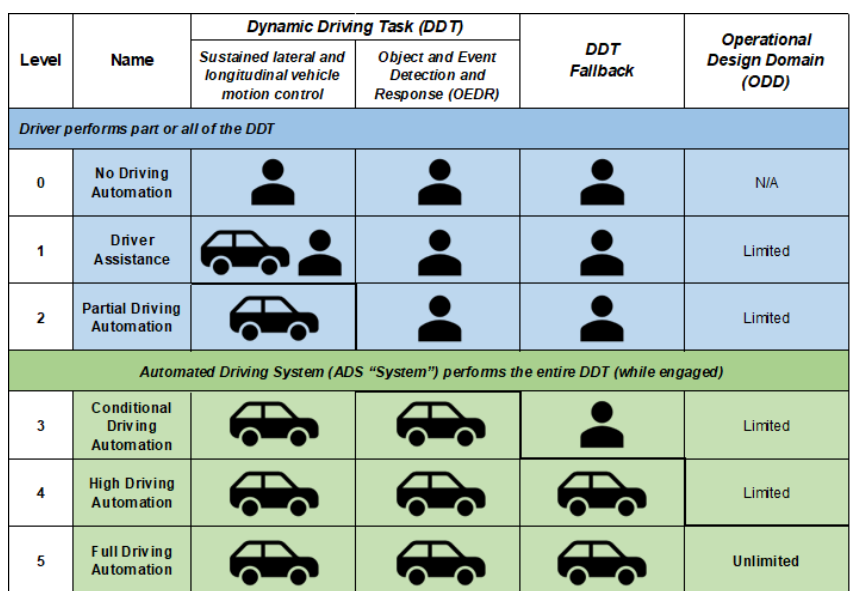

Figure 2: J3016 Standard for autonomous vehicles

\section{EMBEDDED CONTROL IN AUTONOMOUS VEHICLES}

Autonomous control is implemented as embedded control software in a vehicle. This control software codes are written mainly to control both longitudinal and lateral maneuvers of the vehicle. The control algorithm in both maneuvers includes a higher level strategic control and a lower level elementary vehicle control. The strategy control involves decisions based on information from all vehicles affected by the maneuver and the infrastructure. The lower level vehicle control involves control of vehicle steering, throttle and brake systems.

While performing these control, automated vehicles need to incorporate human factors into consideration to restrict vehicle from taking unexpected jerks or motion. Such consideration was taken are key when designing control algorithms for any vehicle maneuver like lane change [4].

\section{A. Longitudinal Control}

Four types of information are necessary for longitudinal control: Speed and acceleration of the host vehicle, the distance to forward vehicle, the speed and acceleration of the preceding vehicle and in case of platoon speed and acceleration of the first vehicle. The speed and acceleration of the host vehicle can be measured by speed sensors and accelerometers (On-board OEM vehicle sensors). The distance to preceding vehicle can be measured using range sensors like LIDAR, vision, radar, and ultrasonic. Radar, has been the most common range sensor in this case [5]. There are two ways to measure speed and acceleration of the preceding vehicle. One way is to derive it from the host vehicle and the measurement from the range sensors.

Another way to obtain the speed and acceleration of the preceding vehicle is by communicating this information between the vehicles [6]. The same method can be used in platooning i.e. the speed and acceleration of the lead (first) vehicle of the platoon are transmitted to vehicles in the platoon. It should be noted that the communication reliability cannot be completely trusted [7].

\section{B. Lateral Control}

The strategic level evaluates the environment for lane change maneuver, like the presence of vehicles in the current and adjacent lane and their dynamics. A strategic level model called MOBIL - Minimizing Overall Braking Induced Lane changes, was proposed to deduct lane changing rules for any optional and mandatory lane changes for different car following models [8]. Different lane change trajectories (circular, the cosine approximation to the circular, the polynomial, and the trapezoidal acceleration) were studied, among them trapezoidal acceleration trajectory was the most desirable for best transition time and passenger's comfort [4]. Two different approaches are presented at the vehicle control level [9]. One approach is to treat the maneuvers as a tracking control problem, another approach uses the unified lateral guidance algorithm. In tracking control, a virtual desired trajectory is generated considering the lateral acceleration and jerk using a sliding mode controller. As for unified lateral guidance approach, a yaw rate generator generates the desired yaw rate for a desired maneuver, either lane change or lane following maneuvers. Commands for steering angle are generated using a reference yaw rate signal and a yaw rate controller for the lane change [10].

\section{Highway Lane Merge}

Highway lane merge problem addressed for connected and automated vehicles, involves both longitudinal and lateral embedded control where the longitudinal takes care of acceleration and braking and the lateral control take care of steering the vehicle during the merge process. A typical highway lane merge maneuver for connected vehicles is shown in the Figure 3. A merging vehicle will attempt to merge from a side lane to the main highway lane usually after finding a gap between the lead and trail vehicles. In a connected architecture, the decision of which vehicle takes the role of lead and trail vehicle on the highway is either decided by the control algorithm externally executed by a road side unit assisting the merge or a de-centralized control algorithm among the connected cars involved in the lane merge maneuver.

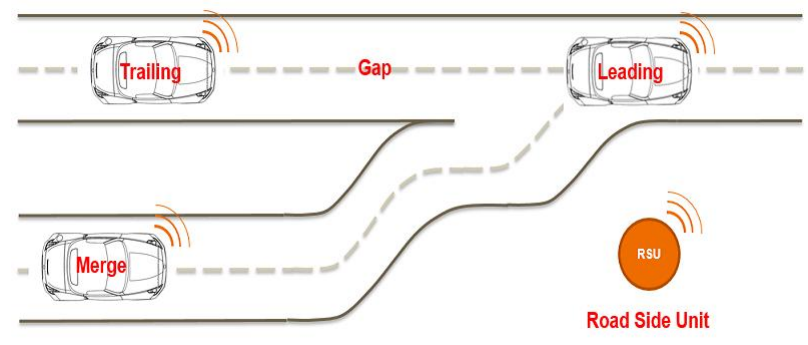

Figure 3: Lane Merge Scenario

Different types of the highway lane merge in the United States are shown in Figure 4 which include the parallel lane merge, tapered lane merge and auxiliary cloverleaf merge. The riskiest of highway merge situation is the tapered acceleration lane as shown in the middle configuration. The current problem is formulated to address this extreme situation using a model predictive optimizing control and will 
eventually be used to solve the parallel acceleration highway lane merge situation.

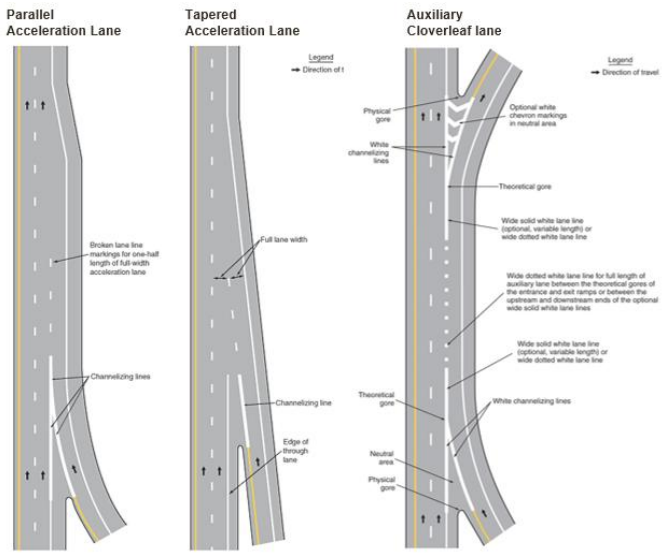

Figure 4: Types of Highway merge

\section{A. Optimization based lane merge}

In a model predictive control optimization, an objective cost function is computed which is a function of the states predicted by a given model and minimized at each sampling time step, starting with the current state and solved over a finite horizon.

The optimal input signal obtained during this optimization is applied to the process only during the following sampling interval. New states are then derived and at the next time-step the optimization problem based on these new measurements or states as initial values is solved again for the shifted horizon. A typical optimal solution is based on the dynamic model of the process, will respects input and output constraints, and minimizes a performance index. The following sub-sections discusses assumptions and modeling realization of the lane merge process, cost functions used for minimization and constraints applied in obtaining the optimal solution.

\section{B. Modelling assumptions}

The mathematical model that is derived is formulated from the merge vehicle perspective. The vehicle system state vector $z_{m} \Xi_{m}$ describes the position and speed of the merge vehicles respectively. At the starting time interval $\boldsymbol{t}_{\mathbf{0}}$, estimates of position and speed of the merge vehicle are made by the on-board sensors or transmitted from other sensors through a V2X communication between vehicle and infrastructure. Using these available states of the merge vehicle as initial conditions, the optimization-based controller will use a kinematic model to predict the future states for a time horizon $\boldsymbol{T}_{\mathbf{p}}$. The optimal control input is determined to optimize criterion or cost which determines the unique path and the secondary controller will steer/accelerate/decelerate based on the optimal path. As mentioned earlier, in a receding implementation, only the first sample of the control input is executed by the actuators and the optimization is repeated by initiating the optimization algorithm with new initial conditions. The assumptions critical to the implementation are as follows:

- All merge vehicles use on-board sensors and sensor fusion to identify merge vehicle`s position and speed within the merging zone.
- The optimization will predict the future evolution of the vehicle system based on a kinematic model which need not be accurate.

- The optimization will make control decisions of steering and acceleration to fulfill the control objectives and constraints.

The optimization objective is subjected to the model behavior and the selection of the model can simplify the optimization formulation or make it more complex in nature. A balance is achieved when considering the complexity of the objective function, constraints, and computation effort available to the designer of the optimal control.

Although kinematics model describes the motion without considering masses or the forces that act on bodies, their use in the optimization problem helps in building simpler formulation. For vehicle dynamics, the bicycle model [11] is extensively used in the merge problem to extract state trajectories. In this model, the velocity vector is computed at the center of mass at $\boldsymbol{l}_{\mathbf{f}}$ from the front and $\boldsymbol{l}_{\mathbf{r}}$ from the back. Where $\boldsymbol{\psi}$ is the yaw angle of the vehicle and $\boldsymbol{\delta}_{\mathrm{f}} \delta_{f}$ is the steering angle of the front wheel. Using the above variable, equations (1)-(5) describe the bicycle model.

$$
\begin{gathered}
\dot{x}=v \cdot \cos (\psi+\beta) \\
\dot{y}=v \cdot \sin (\psi+\beta) \\
\dot{\psi}=\frac{v}{l_{r}} \cdot \sin (\beta) \\
\dot{v}=a
\end{gathered}
$$

where

$$
\beta=\tan ^{-1}\left(\frac{l_{r}}{l_{r}+l_{f}} \cdot \tan \left(\delta_{f}\right)\right)
$$

The states of the merging vehicle model are described by $z_{m}$ $=\left[\begin{array}{llll}x_{\mathrm{m}} & y_{\mathrm{m}} & \psi_{m} & v_{m}\end{array}\right]^{\prime}$ and the input to the model are $\boldsymbol{u}_{\mathrm{m}}=\left[\boldsymbol{a}_{\mathrm{m}}\right.$ $\boldsymbol{\delta}_{\mathbf{f}, \mathbf{m}}$ ]'. The merge vehicle is required to track a reference path while respecting constraints. The complete objective function and the constraints are defined as follows

\section{Objective Function}

Many approaches to formulation of the objective function has been used ranging from simple time optimization to multi-objective functional optimization. The simplest case of objective function in lane merge is the time travel to achieve the merge on the highway [12], [13].

A more generalized multi-objective cost function is formulated for this highway lane merge as a receding horizon control problem minimized over a horizon $\boldsymbol{H}$ for a fixed number of discretized time intervals $\boldsymbol{T}$ for each is shown in eqn. (6)

$$
\min _{u} \sum_{k=1}^{H} \int_{k_{0}+k T}^{k_{0}+T+k T}\left[w^{x} \cdot F_{s}+w^{u} \cdot F_{u}+w^{s} \cdot F_{\text {udiff }}\right]
$$

where

$$
\begin{gathered}
F_{s}=\left(z_{m}-z_{\text {ref }}\right)^{2} \\
F_{u}=u_{m}^{2} \\
F_{u_{-} \text {diff }}=\left(u_{k}-u_{k-1}\right)^{2}
\end{gathered}
$$


and $\boldsymbol{w}^{\boldsymbol{x}}, \boldsymbol{w}^{\boldsymbol{u}}$ and $\boldsymbol{w}^{\boldsymbol{s}}$ are the weights associated with the cost of the sub-objective functions where each function describes a certain cost criterion. These criteria can be explained as below

- Minimizing the error between the desired reference state and actual state (eqn. (7)). States usually include velocities or accelerations of the merge vehicle involved

- Minimizing the control effort, both longitudinal and lateral control inputs (eqn. (8)).

- Minimizing control effort variations to perform a smooth merge maneuver (eqn. (9)).

For the reference trajectory, various displacement trajectories can be used. The profile can be a linear [14] profile or non-linear, like parabolic displacement profile constraint [14]-[16]. For the current optimization formulation two different profiles as described by equation (10) and (11) were considered. Equation (10) is a combination of two linear lines coupled over with a sigmoid function. The sigmoid function allows the smooth transition from one linear profile to the other using the parameter $\boldsymbol{\alpha}$.

$$
y=(m x+c)+\left[\frac{1+\tanh (\alpha(x-c))}{2}\left(\frac{w}{2}-(m x+c)\right)\right]
$$

Another reference profile that is used is obtained using similar parameters as described by [15] and given by the equation (11).

$$
y=\frac{m}{2} \cdot\left[(x-c)+\left((x-c)^{2}-\frac{\alpha}{m}\right)^{2}\right]
$$

\section{Constraints for merge optimization.}

The optimization will find inputs that minimize the objective function which is the function of states and inputs of the lane merge process. During the lane merge problem, inputs to the vehicles are limited and it is usually desired that the states follow certain limits. Most constraints limit the states and inputs and their rates. And in most cases a set of linear inequalities defines the bounds. The following are constraints used in this highway merge optimization.

The inputs to the kinematic model are acceleration and steering. Both inputs, accelerations and steering are bounded by the maximum and minimum values defined by the vehicle parameters. In most cases, the throttle and braking constraints are combined to limit the acceleration and deceleration of the vehicle as one bounded input. A general constraint for this highway merge for all inputs is given as follows

$$
\begin{gathered}
-u_{\text {min }}(t) \leq u(t) \leq u_{\text {max }}(t) \ldots \forall t \\
-\delta_{f, \text { min }}(t) \leq \delta_{f}(t) \leq \delta_{f, \text { max }}(t) \ldots \forall t
\end{gathered}
$$

It is recommended practice in applied control to constraint the rate of change of the input. Like most problem formulation, wherein acceleration is the main input parameter to highway merge problem, the rate of change of acceleration is considered as jerk experienced by the vehicle. Using this constraint improves the passengers experience in the vehicle and smoothen the application of the input during the lane merge. It is expressed by eqn. (14) as follows

$$
-\dot{u}_{\min }(t) \leq \dot{u}(t) \leq \dot{u}_{\max }(t) \ldots \forall t
$$

Steering angle for lateral movement of the vehicle is also constrained in the current formulation, as considered in the sliding mode controller [14]. Using a similar approach, rate of lateral movement of the vehicle is limited which depends on the steering input, which can be defined as below

$$
-\dot{\delta}_{f, \min }(t) \leq \dot{\delta}_{f}(t) \leq \dot{\delta}_{f, \max }(t) \ldots \forall t
$$

Velocity constraints are also common state constraint that is applied for all formulations of merge problem. The vehicles involved in the merge process should have the velocities bounded within the minimum and maximum allowable limits. This limits the optimal control to produce results that govern the limits on the merging lane and the main lane. A generalized velocity constraint is shown below

$$
-v_{\min }(t) \leq v(t) \leq v_{\max (t)}(t) \ldots \forall t
$$

\section{Simulation And Results}

A numerical solution is presented where the simulations were conducted using Matlab ${ }^{\circ}$ and its optimization toolbox using the default interior-point algorithm for solving non-linear formulation. Reports were generated on the computation load encountered during the optimization problem.

The results of the optimization are discussed as follows. The first result is obtained over a receding horizon of 1.6 seconds using eqn. (10) as the merging lane profile starting the optimization with an initial condition of $z_{m, t=0}=\left[\begin{array}{llll}0 & -10 & 45 & 10\end{array}\right]$. The optimized first input is used to move one time-step ahead and the new states are used as new initial conditions and the optimization is repeated to get a new optimization result. The constraints on the system were the limitation to acceleration and steering given by $u_{l b}=\left[\begin{array}{ll}-5 & -50\end{array}\right]$ and $u_{u b}=\left[\begin{array}{ll}5 & 50\end{array}\right]$ respectively which limits the acceleration/braking to $5 \mathrm{~m} / \mathrm{s}^{2}$ and steering to $50^{\circ}$. The result is shown in figure (5) which shows that the merge vehicle can track the merge lane profile while respecting the input limits.

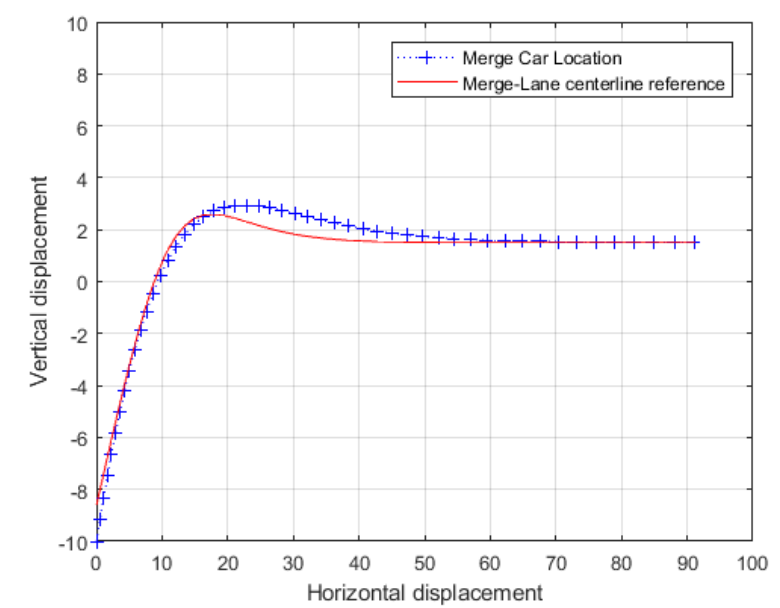

Figure 5: Optimized results for Highway Merge vehicle using equation (10) 
Another simulation was performed with equation (11) as a reference profile trajectory. The initial conditions and constraints are similar to the previous results with an addition of velocity constraint as described by equation (16). In order to prevent backing up the velocity constraints on optimization was limited between $0 \mathrm{~m} / \mathrm{s}$ and $10 \mathrm{~m} / \mathrm{s}$.
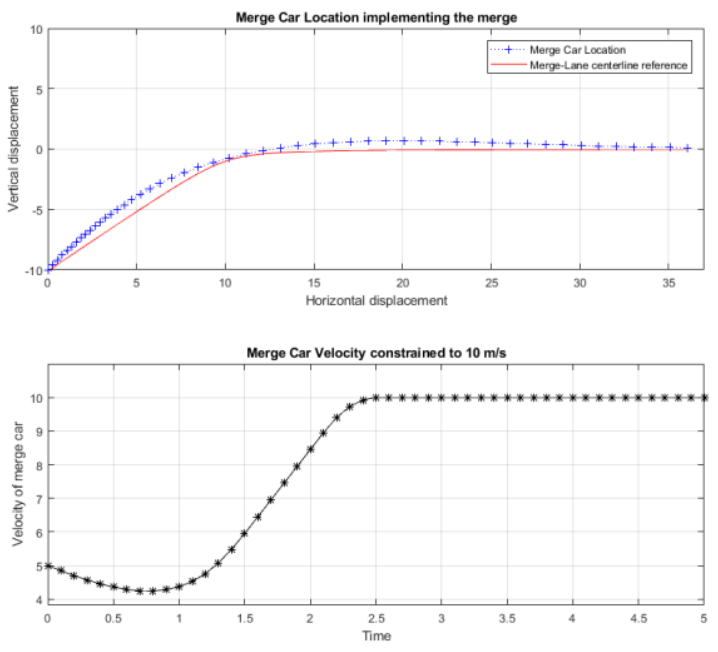

Figure 6: Optimized results for highway merge vehicle using reference equation (11)

The simulation results show that the merge car follows the merge lane profile while respecting the bounds on the input and the velocity constraint. The inputs to the merge vehicle is given in the next figure.
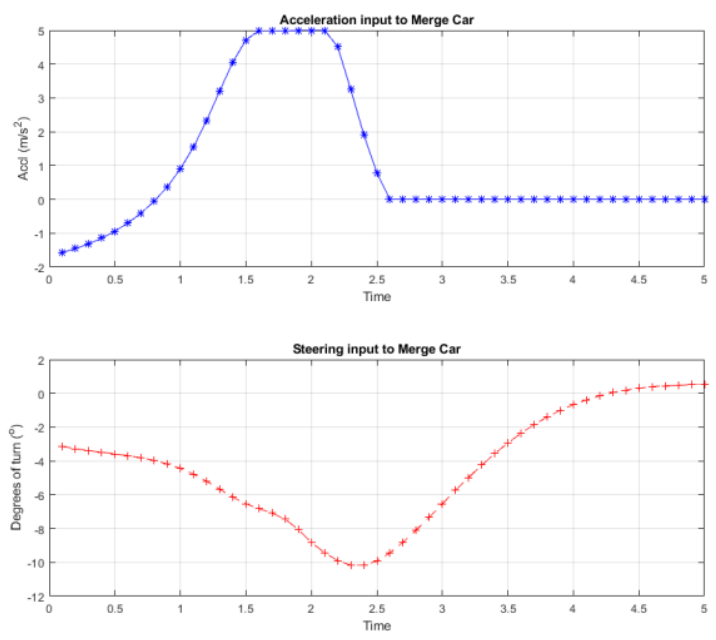

Figure 7: Constrained inputs of the merge vehicle

Results are computed on a Windows 10 machine with an Intel ${ }^{\circledR}$ Core 2 Duo with $3.0 \mathrm{GHz}$ processor speed and eight $\mathrm{Gb}$ of memory has an average time of 1.2 seconds. A very fast generalized minimum residual method (GMRES) method was used to obtain the solution of an associated state-dependent two-point boundary-value problem which was the case for [16], can be explored but not used to get the above results. For current optimization algorithms used in obtaining the results, the complexity of implementing the online real-time optimization can have less impact as increasingly powerful platforms are available to solve it.

\section{CONCLUSION}

The highway lane merge for merge lane without the acceleration area are seen in many highway merge situations. It is important that any autonomous vehicle should be able to navigate through such maneuvers with ease. An MPC based optimization method for such a maneuver was implemented subjected to the constraints encountered by the autonomous system.

Implementation of a single merge car on a local lane was presented, but an autonomous co-operative merge into a gap between a lead car and trail car, selected from a set of cars on the highway lane, is currently being explored and will be scope of future research work.

\section{REFERENCES}

[1] K. Bengler, K. Dietmayer, M. Maurer, and H. Winner, "Three Decades of Driver Assistance Systems Review and Future Perspectives," no. October, pp. 6-22, 2014.

[2] US Department of Transportation and Safety, "Federal Automated Vehicles Policy: Accelerating the Next Revolution In Roadway Safety," 2016.

[3] U. department of transportation Safety, "Automated Driving Systems: A Vision for Safety 2.0," US Department of Transportation, 2017.

[4] W. Chee and M. Tomizuka, "Vehicle Lane Change Maneuver in Automated Highway Systems," Calif. Partners Adv. Transit Highw., no. Lc, 1994.

[5] H. H. Meinel, "Commercial Applications of Millimeterwaves History, Present Status, and Future Trends," IEEE Trans. Microw. Theory Tech., vol. 43, no. 7, pp. 1639-1653, 1995.

[6] R. Rajamani and S. . Shladover, "An experimental comparative study of autonomous and co-operative vehicle-follower control systems," Transp. Res. Part C Emerg. Technol., vol. 9, no. 1, pp. 15-31, 2001.

[7] R. Rajamani, H. S. Tan, B. K. Law, and W. Bin Zhang, "Demonstration of integrated longitudinal and lateral control for the operation of automated vehicles in platoons," IEEE Trans. Control Syst. Technol., vol. 8, no. 4, pp. 695-708, 2000.

[8] A. Kesting, M. Treiber, and D. Helbing, "General Lane-Changing Model MOBIL for Car-Following Models," Transp. Res. Rec. J. Transp. Res. Board, vol. 1999, no. 1, pp. 86-94, 2007.

[9] W. Chee, "Unified Lateral Control System for Intelligent Vehicles," SAE Tech. Pap. Ser., no. 724, 2000.

[10] C. Hatipoglu, U. Ozguner, and K. A. Redmill, "Automated lane change controller design," IEEE Trans. Intell. Transp. Syst., vol. 4, no. 1, pp. 13-22, 2003.

[11] R. Rajamani, Vehicle Dynamics and Control. Boston, MA: Springer US, 2012.

[12] G. Raravi and V. Shingde, "Merge algorithms for intelligent vehicles," Next Gener. Des. ..., pp. 51-65, 2007.

[13] T. Awal, L. Kulik, and K. Ramamohanrao, "Optimal traffic merging strategy for communication- and sensor-enabled vehicles," in IEEE Conference on Intelligent Transportation Systems, Proceedings, ITSC, 2013, pp. 1468-1474.

[14] P. Kachroo and Z. L. Z. Li, "Vehicle merging control design for an automated highway system," Proc. Conf. Intell. Transp. Syst., no. 1, pp. 224-229, 1997.

[15] W. Cao, M. Mukai, and T. Kawabe, "Two-dimensional merging path generation using model predictive control," Artif. Life Robot., vol. 17, no. 3-4, pp. 350-356, 2013.

[16] W. Cao, M. Mukai, T. Kawabe, H. Nishira, and N. Fujiki, "Gap Selection and Path Generation during Merging Maneuver of Automobile Using Real-Time Optimization," SICE J. Control. Meas. Syst. Integr., vol. 7, no. 4, pp. 227-236, 2014.

Syed A. Hussain is currently pursuing Ph.D. in Electrical and Computer Engineering at University of Illinois at Chicago, Chicago, Illinois since 2014. His research interest is in applied mechatronics engineering with special interest in autonomous vehicles engineering. He also worked as a mechatronics intern for a summer at Servotech working on developing hardware-in-loop testing platform.

Earlier to his current studies, he was a lecturer in King Fahd University of Petroleum and Mineral teaching controls and instrumentation from 2014. He obtained master's degree from Systems Engineering, King Fahd University of Petroleum and Minerals (K.F.U.P.M.) in Controls and Instrumentation 
and has a Bachelor of Mechanical Engineering at Muffakham Jah College of Engineering (M.J.C.E.T.), Osmania University, Hyderabad, India.

Babak Shahian-Jahromi is currently a Ph.D. Candidate in Mechanical Engineering at University of Illinois at Chicago since 2014. Earlier to his current studies, he received his Master of Science degree in Mechanical Engineering, from University of Illinois at Chicago (UIC) and a Bachelor of Science degree in Mechatronics Engineering at Sharif University of Technology (SUT), Tehran, Iran. His research interest is in applied Mechatronics engineering with a focus on autonomous vehicles engineering.

Burak Karakas is currently pursuing Ph.D. in Mechanical Engineering at University of Illinois at Chicago, Chicago, Illinois since 2016. His research interest is in automotive engineering with special interest in autonomous vehicles engineering. Earlier to his current studies, He obtained a Master's degree from Mechanical Engineering, at University of Illinois at Chicago and has a Bachelor of Science degree in Automotive Engineering at Kocaeli University, Kocaeli, Turkey.

Sabri Cetin is a full Professor at University of Illinois at Chicago (UIC). $\mathrm{He}$ got his Ph.D. in Mechanical Engineering from Georgia Institute of Technology (1987) and worked at UIC since. Previously, he obtained his Master of Science from Georgia Institute of Technology (1984) and bachelor's in aerospace engineering from Technical University of Istanbul (1982). He has received many honors during his tenure at UIC including the UIC faculty research award. He has filed five patents between 2001 and 2007. His major research interest includes mechatronics and control systems.

$\mathrm{He}$ is currently the director of the Mechatronics Laboratory and focuses on various aspects of motion control of mechanical systems including, DC servo motor control, ultra-precision motion control using novel piezoelectric actuators designed in our lab, electro-hydraulic servo control with earth moving equipment applications, adaptive self-learning real time control algorithms. 